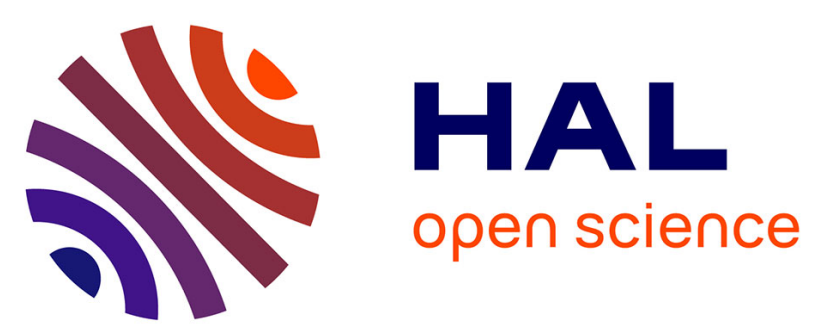

\title{
Effectiveness Evaluation of Arm Usage for Human Quiet Standing Balance Recovery through Nonlinear Model Predictive Control
}

Keli Shen, Ahmed Chemori, Mitsuhiro Hayashibe

\section{- To cite this version:}

Keli Shen, Ahmed Chemori, Mitsuhiro Hayashibe. Effectiveness Evaluation of Arm Usage for Human Quiet Standing Balance Recovery through Nonlinear Model Predictive Control. ICCR 2020 - 3rd International Conference on Control and Robots, Dec 2020, Tokyo, Japan. pp.150-153, 10.1109/ICCR51572.2020.9344184 . lirmm-03131502

\section{HAL Id: lirmm-03131502 \\ https://hal-lirmm.ccsd.cnrs.fr/lirmm-03131502}

Submitted on 4 Feb 2021

HAL is a multi-disciplinary open access archive for the deposit and dissemination of scientific research documents, whether they are published or not. The documents may come from teaching and research institutions in France or abroad, or from public or private research centers.
L'archive ouverte pluridisciplinaire HAL, est destinée au dépôt et à la diffusion de documents scientifiques de niveau recherche, publiés ou non, émanant des établissements d'enseignement et de recherche français ou étrangers, des laboratoires publics ou privés. 


\section{Effectiveness Evaluation of Arm Usage for Human Quiet Standing Balance Recovery through Nonlinear Model Predictive Control}

\author{
Keli Shen \\ Department of Robotics \\ Tohoku University \\ Sendai, Japan \\ Email: shen.keli.s3@dc.tohoku.ac.jp
}

\author{
Ahmed Chemori \\ LIRMM, CNRS \\ University of Montpellier \\ Montpellier, France \\ Email: Ahmed.Chemori@lirmm.fr
}

\author{
Mitsuhiro Hayashibe \\ Department of Robotics \\ Tohoku University \\ Sendai, Japan \\ Email: hayashibe@tohoku.ac.jp
}

\begin{abstract}
The computational study of human balance recovery strategy is crucial for revealing effective strategy in human balance rehabilitation and humanoid robot balance control. In this context, many efforts have been made to improve the ability of quiet standing human balance. There are three main strategies for human balance including (i) ankle, (ii) hip, and (iii) stepping strategies. Besides, arm usage was considered for balance control of human walking. However, there exist few works about effectiveness assessment of arm strategy for quiet standing balance recovery. In this paper, we proposed a nonlinear model predictive control (NMPC) for human balance control on a simplified model with sagittal arm rotation. Three case studies including ( i) active arms, (ii) passive arms, and (iii) fixed arms were considered to discuss the effectiveness of arm usage for human balance recovery during quiet standing. Besides, the total root mean square (RMS) deviation of joint angles was computed as an index of human motion intensity quantification. The proposed solution has been implemented for a human-like balance recovery with arm usages during quiet standing under perturbation and shows the effectiveness of arm strategy.

Keywords-NMPC, RMS deviation, effectiveness evaluation, arm rotating.
\end{abstract}

\section{INTRODUCTION}

Human balance control has been studied for many years [1]. There are three main balance recovery strategies, including ankle strategy, hip strategy, and stepping strategy, which have been studied from human experiments [2], [3], [4] and artificial systems [5], [6], [7], [8]. These strategies have been qualified as the most efficient ways to help preventing falls and to understand the mechanism of balance control during standing and walking [9] in human rehabilitation and robotic applications. In addition to the above mentioned balance strategies, arm strategy has also been considered as an efficient way to contribute to balance control and reduce fall impacts as well [10], [11].

Various interesting works related to arm strategy have been proposed and validated through human experiments and simulations. Nashner et al. [12] have tested rapid postural adjustment associated with a class of voluntary movements, including arm rotation that disturbs the postural balance. The study [13] suggested that maximization of gait efficiency based on an organism's propensity is considered for the convergence towards the stable coordination between arms and legs. Atkeson et al. [14] proposed an optimal control from one optimization criterion to implement a human-like balance recovery on a multi-link model and observed the shoulder rotations for different perturbations. Nakada et al. [15] proposed a Q-learning to produce appropriate arm control torques and concluded that the arm rotation strategy enlarges the manageable range of perturbation impulses.

After reviewing previous works on this topic, we found that they did not cover the verification of arm strategy with multi-constraints: active arms, passive arms, and fixed arms in their human experiments. They neither took advantage of nonlinear model predictive control (NMPC) for dealing with constraints on angles and torques of the ankle, hip, and arm joints, nor optimizing human-like balance recovery movements in their artificial systems. Consequently, we further developed the control mechanism of arm strategy for balance recovery based on the previous works. The main contributions of the present paper can be summarized as follows.

1) We built a five-link, three-joint model to represent the human body structure for a quiet standing balance recovery study in A/P direction.

2) NMPC with input and state constraints is proposed and implemented for human-like balance behavior. Three different arm states are considered to study the effectiveness of arm strategy for balance recovery: (i) active arms, (ii) passive arms, and (iii) fixed arms.

3) Different amplitudes of disturbing forces are considered and applied to the model to observe the behavior of the system under the proposed NMPC and arm strategy.

4) A root mean square (RMS) performance index based on joint angles is proposed for human motion intensity quantification for the first time. By comparing the resulting RMS deviation values in different arm states, it was possible to conclude that balance recovery with active arms is the most effective way, and balance control with arms usage is better than without arms usage.

\section{Description of the Simplified Human Model}

To implement the quiet standing balance recovery, we consider the human body as a five-link model comprising a fixed foot, an ankle joint, a lower body, a hip joint, an upper body, a left-right arm joint, a right arm, and a left arm, as illustrated in Fig. 1. The physical parameters of this model are summarized in TABLE I. Based on an existing anthropometric database [16], the total body height is $1.7[\mathrm{~m}]$ and the total body mass is 
TABLE I

SUMMARY OF THE PHYSICAL PARAMETERS OF THE PROPOSED FIVE-LINK, THREE-JOINT MODEL.

\begin{tabular}{|c|c|c|c|}
\hline Link & Mass $[\mathrm{kg}]$ & Length $[\mathrm{m}]$ & Height $[\mathrm{m}]$ \\
\hline Foot & 1.3 & 0.3 & 0.1 \\
\hline Lower body & 35 & 1.0 & - \\
\hline Upper body & 25 & 0.6 & - \\
\hline Right arm & 4 & 0.6 & - \\
\hline Left arm & 4 & 0.6 & - \\
\hline Total weight $[\mathrm{kg}]$ & 69.3 & - & - \\
\hline Total height $[\mathrm{m}]$ & - & - & 1.7 \\
\hline
\end{tabular}

$69.3[\mathrm{~kg}]$. It is worth noting that we ignore the body segments between the ankle joint and the hip joint, between the hip joint and the left-right arm joint, and between the left-right arm joint and the head. This is consistent with the case of human quiet standing balance, because humans maintain their knee joint angle within a certain range of disturbing forces acting on their body. However, if these disturbing forces become too large, they need to use their other body joints including knee, waist, neck joints, etc., and step forward to avoid falling down.

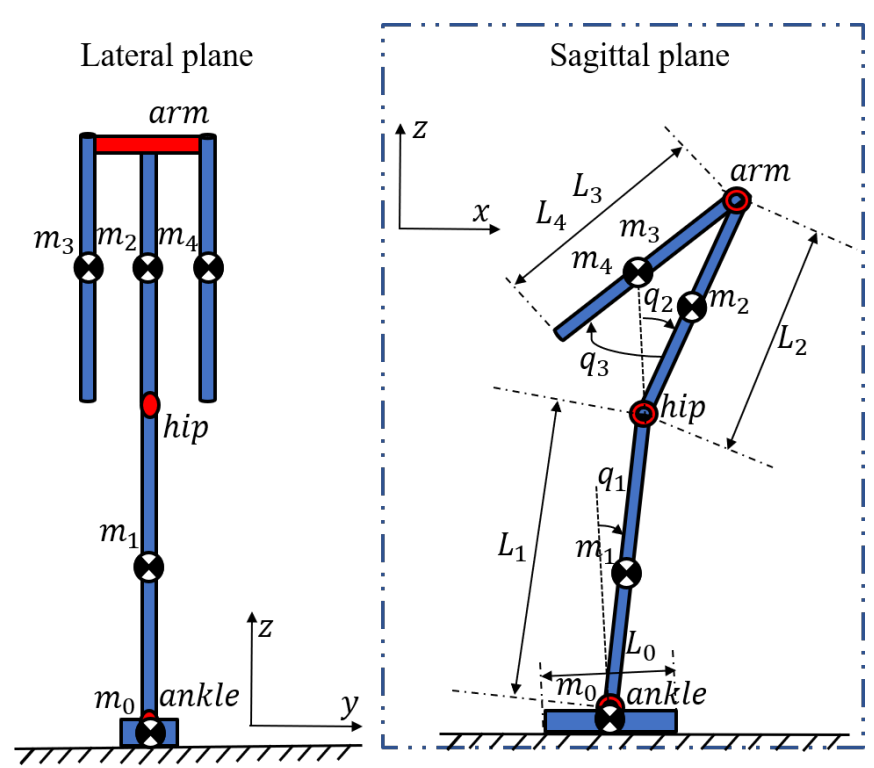

Fig. 1. Structure of the five-link, three-joint model. $m_{0}, m_{1}, m_{2}, m_{3}, m_{4}$ represent the masses of foot, lower body, upper body, right arm and left arm respectively. $L_{0}, L_{1}, L_{2}, L_{3}, L_{4}$ represent the lengths of foot, lower body, upper body, right arm, and left arm, respectively. $q_{1}, q_{2}, q_{3}$ represent the ankle join angle, hip joint angle, left-right arm joint angle, respectively. The right and left arms share the same joint motor.

\section{DyNAMIC EQUATIONS OF THE Simplified Model}

Lagrange formalism [17] is applied to derive the dynamic equation of motion for this five-link, three-joint model controlled by the ankle, hip, and arm joint-torques. The Lagrange equations and dynamic equation of motion are separately derived for the model with three different arm states including (i) active arms, (ii) passive arms, and (iii) fixed arms.

The dynamic equations of the model with active arms should consider the rotation of arm joint in the sagittal plane under the control torques generated by the joint actuator. The Lagrange equations for this case are as follows:

$$
\begin{aligned}
\frac{d}{d t}\left(\frac{\partial L}{\partial \dot{q}_{1}}\right)-\frac{\partial L}{\partial q_{1}} & =\tau_{\text {ankle }} \\
\frac{d}{d t}\left(\frac{\partial L}{\partial \dot{q}_{2}}\right)-\frac{\partial L}{\partial q_{2}} & =\tau_{\text {hip }} \\
\frac{d}{d t}\left(\frac{\partial L}{\partial \dot{q}_{3}}\right)-\frac{\partial L}{\partial q_{3}} & =\tau_{\text {arm }} \\
L & =T-V .
\end{aligned}
$$

Where, $L$ is the Lagrangian function, $T$ is the total kinetic energy, $V$ is the total potential energy, $\tau_{\text {ankle }}$ is the ankle torque, $\tau_{\text {hip }}$ is the hip torque, and $\tau_{\text {arm }}$ is the arm torque.

The resulting dynamic equation of motion can be expressed in a matrix form as follows:

$$
\left[\begin{array}{lll}
M_{11} & M_{12} & M_{13} \\
M_{21} & M_{22} & M_{23} \\
M_{31} & M_{32} & M_{33}
\end{array}\right]\left[\begin{array}{l}
\ddot{q}_{1} \\
\ddot{q}_{2} \\
\ddot{q}_{3}
\end{array}\right]+\left[\begin{array}{c}
C_{1} \\
C_{2} \\
C_{3}
\end{array}\right]=\left[\begin{array}{c}
\tau_{\text {ankle }} \\
\tau_{\text {hip }} \\
\tau_{\text {arm }}
\end{array}\right] .
$$

In (5), $M_{11}, M_{22}$ and $M_{33}$ are the effective inertia terms, $M_{12}$, $M_{13}, M_{21}, M_{23}, M_{31}$, and $M_{32}$ are the coupling inertia terms. $C_{1} C_{2}$, and $C_{3}$ terms include centrifugal, Coriolis, and gravity forces.

Here, the derivation of the dynamic equations of the model for the cases with passive arms and fixed arms is omitted. The important remarks for the different conditions of derivation are given as follows. In dynamic equations of the model with passive arms, there is no control torque of the arm joint (i.e., $\tau_{\text {arm }}=0$ ) in the dynamic equation (3) due to the passive arm state. In dynamic equations of model with fixed arms, there is no control torque, nor rotation of the arm joint in the dynamic equation due to the fixed arm state. Consequently, equation (3) can be omitted.

\section{Proposed NMPC For BALANCE RECOVERY}

In this section, we propose an NMPC scheme [18], [19], [20] to resolve balance recovery problem. The NMPC problem described above can be solved as an iterative open-loop optimal control problem with a finite horizon and an observable initial state for each sampling time.

The cost function considered in the optimal control problem of NMPC is

$$
J\left(\boldsymbol{x}(0), \boldsymbol{\tau}_{(0, N-1)}\right)=\sum_{0}^{N-1} l(\boldsymbol{x}, k, \boldsymbol{\tau})+V_{f},
$$

$$
\begin{aligned}
& l(\boldsymbol{x}, k, \boldsymbol{\tau})=\frac{1}{2}\left(\boldsymbol{x}^{T}(k) \boldsymbol{Q} \boldsymbol{x}(k)+\boldsymbol{\tau}^{T}(k) \boldsymbol{R} \boldsymbol{\tau}(k)\right), \\
& V_{f}=\frac{1}{2} \boldsymbol{x}^{T}(N) Q_{f} \boldsymbol{x}(N) .
\end{aligned}
$$


The penalty weighting and constraints of NMPC are different for the model with the three different arm states: active arms, passive arms, and fixed arms. The objective is to minimize the cost $J\left(\boldsymbol{x}(0), \boldsymbol{\tau}_{(0, N-1)}\right)$ subject to the state and control constraints:

1) The joint torques should satisfy the input constraints:

$$
\begin{aligned}
-120[\mathrm{Nm}] \leq \tau_{\text {ankle }} & \leq 120[\mathrm{Nm}] \\
-500[\mathrm{Nm}] & \leq \tau_{\text {hip }} \leq 500[\mathrm{Nm}] \\
-200[\mathrm{Nm}] \leq \tau_{\text {arm }} & \leq 200[\mathrm{Nm}] .
\end{aligned}
$$

2) The joint angles satisfy the state constraints:

$$
\begin{aligned}
-0.2[\mathrm{rad}] \leq x_{\text {ankle }} & \leq 0.4[\mathrm{rad}], \\
-0.35[\mathrm{rad}] \leq x_{\text {hip }} & \leq 1.3[\mathrm{rad}], \\
-2.5[\mathrm{rad}] \leq x_{\text {arm }} & \leq 0.5[\mathrm{rad}] .
\end{aligned}
$$

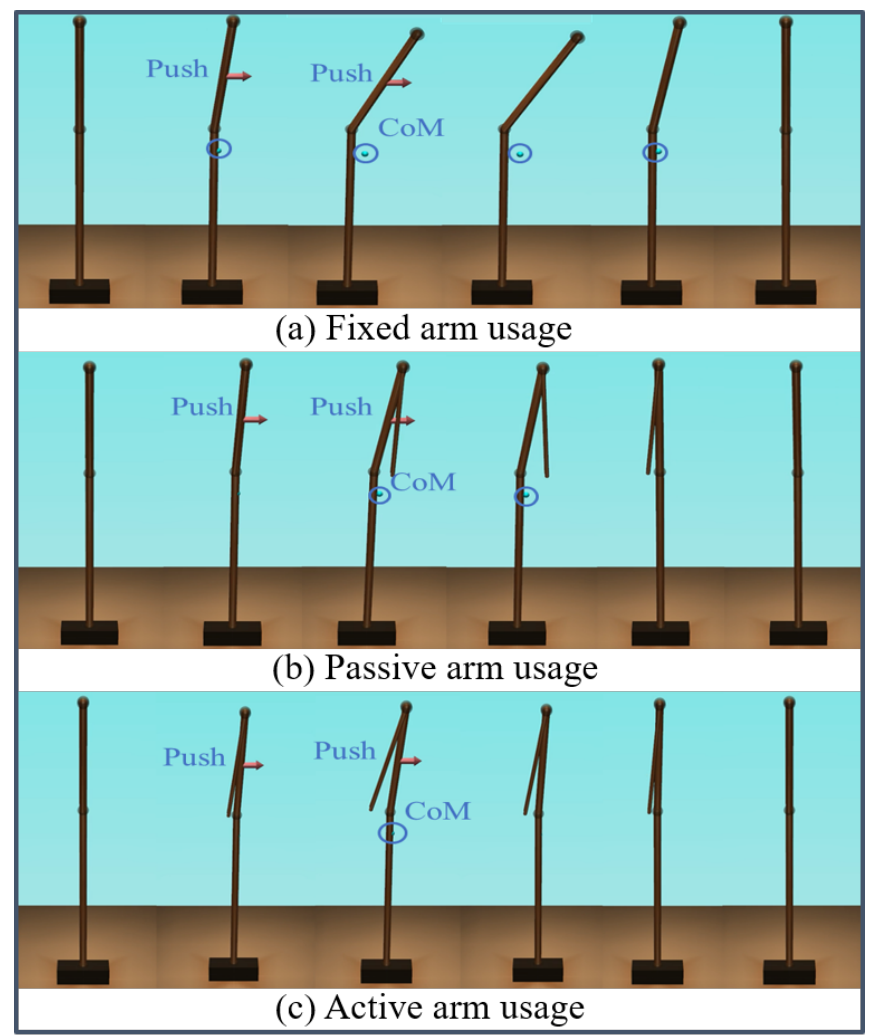

Fig. 2. Screenshots of the balance behavior for the three different arm states for a disturbing force of $70[N]$.

\section{Simulation Results AND Discussion}

In this section, we employ total RMS deviation of the joint angles as an analysis index of the model motion intensity to confirm the effectiveness of arm strategy. The CoM of the upper body is chosen as push position with different disturbing forces for a duration of $1[s]$. The disturbing forces are determined in sagittal plane (backward and frontward directions): $-80[N],-70[N],-60[N],-40[N],-20[N]$,
$0[N], 20[N], 40[N], 60[N], 70[N]$, and $80[N]$. The body can recover balance after the perturbation within a recovery time of $4[s]$. Only the model with active arm usage has ability of controlling balance recovery from the unstable states under the push forces $-80[N]$ and $80[N]$. The models with passive and fixed arm usages are impossible to have an effective solution for balance recovery in this case. This demonstrates that the arm strategy based on active arm rotating enlarges the manageable range of the push forces, within which the balance recovery is controllable. This observation is consistent with the results made in [15].

The screenshots of movements of the models with active, passive, and fixed arm usages under the disturbing force $70[N]$ are shown in Fig. 2. Here, we can observe that the model with the active arm rotation obtains a strong ability to maintain balance recovery than the others, since the deviation of the center of mass in the $\mathrm{x}$-axis of the model with active arm rotating is less than the others. This shows that active arms rotation can help to keep CoM of the body close to the equilibrium point ensuring consequently a better stability.

The total RMS deviation is computed by

$$
\text { Total RMS deviation }=\sqrt{\frac{1}{N} \sum_{t=1}^{N}\left(q_{\text {ankle }}(t)^{2}+q_{\text {hip }}(t)^{2}\right)}
$$

here, $N$ is the simulation sampling number. $q_{\text {ankle }}(t)$, qhip denote the ankle, hip angles at each simulation sampling point respectively.

The Comparison of total RMS deviation of the model under the different push forces with the three, different arm states are depicted in Fig. 3. The total RMS deviation is proposed as an index of the body motion intensity. Fig. 3 shows that total RMS deviation of maintaining balance motion with fixed arm rotation is larger than that with passive arm rotation, and total RMS deviation of maintaining balance motion with passive arm usage is larger than that with active arm usage for the push forces: $-70[N],-60[N],-40[N],-20[N], 20[N]$, $40[N], 60[N], 70[N]$. This illustrates clearly that arm rotations contribute significantly to the human body balance recovery control and reduce the motion intensity of the hip joint. This conclusion is consistent with the results obtained from the human experiments [21].

\section{CONCLUSION}

In this paper, we proposed an NMPC scheme to resolve the problem of human-like balance behavior with arm rotation on a simplified human model. Three arm usages including (i) active arms, (ii) passive arms, and (iii) fixed arms are compared in this study to evaluate the effectiveness of arm usage in quiet standing balance recovery for the different push forces. By comparing an index named the RMS deviation of joint angles, the effectiveness of the arms usage for human balance control is verified, and balance control with active arm usage demonstrates the best performance. In this study, we could reproduce the predictive arm usage by NMPC to compensate the disturbance to the postural balance. By comparison, the 


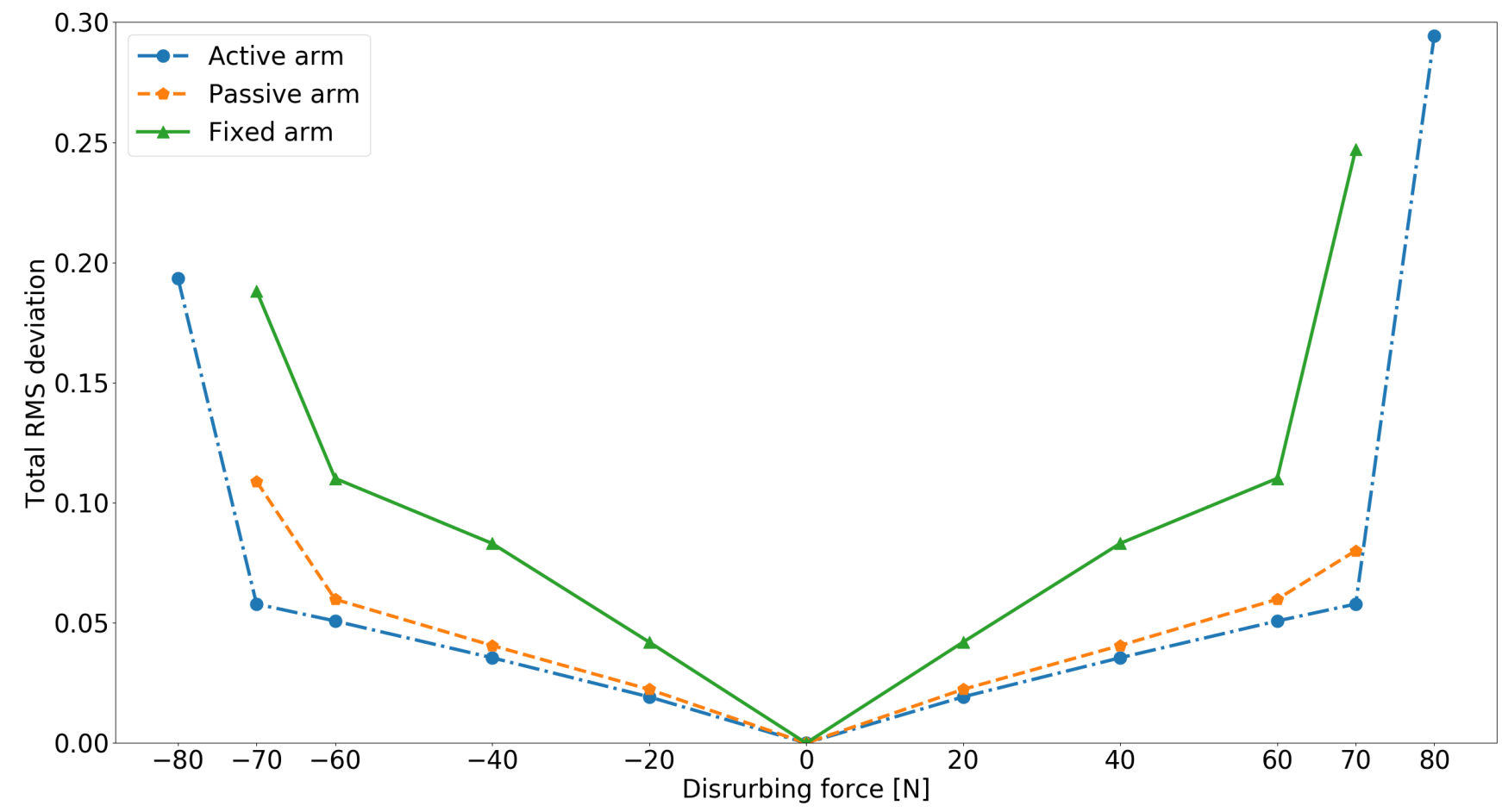

Fig. 3. Comparison of total RMS deviation of the model with three different arm states for the different push forces in backward and frontward direction: $-80[N],-70[N],-60[N],-40[N],-20[N], 0[N], 20[N], 40[N], 60[N], 70[N]$, and $80[N]$.

predictive arm usage demonstrated that it can reduce the burden of balance coordination task, as we can observe human arm strategy in postural control.

\section{ACKNOWLEDGMENT}

This work was supported by the G-7 Scholarship Foundation and the GP-mech Program of Tohoku University, Japan.

\section{REFERENCES}

[1] D. Winter, Human balance and posture control during standing and walking, Gait and Posture, vol. 3, no. 4, pp. 193214, Dec. 1995.

[2] H. Hemami and P. Camana, Nonlinear feedback in simple locomotion systems, IEEE Trans. Automatic Control, vol. 21, no. 6, pp. 855860 , Dec. 1976.

[3] L. M. Nashner, G. McCollum, The organization of human postural movements: a formal basis and experimental synthesis, Behav. Brain Sci, vol. 8, no. 1, pp. 135-150, 1985.

[4] F. B. Horak, L. M. Nashner, and H. C. Diener, Postural strategies associated with somatosensory and vestibular loss, Exp. Brain Res., vol. 82, no. 1, pp. 167-177, 1990.

[5] A. D. Kuo, F. E. Zajac, Human standing posture: multi-joint movement strategies based on biomechanical constraints, Progr. Brain Research, vol. 97, pp. 349-358, Jan, 1993.

[6] A. Kuo, An optimal control model for analyzing human postural balance, IEEE Trans. Biomedical Engineering, vol. 42, pp. 87101, 1995.

[7] S. Okajima, M. Tournier, F. S Alnajjar, M. Hayashibe, Y. Hasegawa, S. Shimoda, Generation of Human-like Movement from Symbolized Information, Frontiers in Neurorobotics, 12:43, 2018.

[8] K. Shen, A. Chemori and M. Hayashibe, Human-Like Balance Recovery Based on Numerical Model Predictive Control Strategy, IEEE Access, vol. 8, pp. 92050-92060, 2020.

[9] D. Galdeano, V. Bonnet, M. Bennehar, P. Fraisse, A. Chemori, Partial Human Data in Design of Human-Like Walking Control in Humanoid Robotics, 10th IFAC Symposium on Robot Control International Federation of Automatic Control, Dubrovnik, Croatia, Sep. 5-7, 2012.
[10] PE. Roos, MP. McGuigan, DG. Kerwin, G. Trewartha, The role of arm movement in early trip recovery in younger and older adults. Gait Posture, vol.27, no. 2, pp. 352-356, 2008.

[11] M. Pijnappels, I. Kingma, D. Wezenberg, G. Reurink, JH. van Dien, Armed against falls: the contribution of arm movements to balance recovery after tripping. Exp Brain Res., vol.201, no. 4, pp. 689-699, 2010.

[12] P. J. Cordo and L. M. Nashner, Properties of postural adjustments associated with rapid arm movements, J. Neurophysiol., vol. 47, no. 2, pp. 287-302, 1982.

[13] A. Ledebt, Changes in arm posture during the early acquisition of walking, Infant Behav. Dev., vol. 23, no. 1, pp. 79-89, 2000.

[14] C. G. Atkeson and B. Stephens, Multiple balance strategies from one optimization criterion, Proc. 2007 7th IEEE-RAS Int. Conf. Humanoid Robot. HUMANOIDS 2007, pp. 57-64, 2007.

[15] M. Nakada, B. Allen, S. Morishima and D. Terzopoulos, Learning Arm Motion Strategies for Balance Recovery of Humanoid Robots, 2010 International Conference on Emerging Security Technologies, Canterbury, pp. 165-170, 2010.

[16] M. Kouchi, M. Mochimaru, H. Iwasawa and S. Mitani, Anthropometric database for Japanese Population 1997-98, Japanese Industrial Standards Center (AIST, MITI), Japan, 2000.

[17] R. P. Paul, Robot Manipulators: Mathematics, Programming and Control, The MIT Press, 1981.

[18] F. Allgwer, A. Zheng, Nonlinear Model Predictive Control, Springer, 2000.

[19] L. Grüne, J. Pannek, Nonlinear Model Predictive Control: Theory and Algorithms, 2nd ed, Springer, Switzerland, 2017.

[20] L. Magni, D. M. Raimondo, F. Allgwer, Nonlinear Model Predictive Control: Towards New Challenging Applications, Springer-Verlag Berlin Heidelberg, 2009.

[21] K. J. Boström, T. Dirksen, K. Zentgraf, and H. Wagner, The contribution of upper body movements to dynamic balance regulation during challenged locomotion, Front. Hum. Neurosci., vol. 12, no. January, pp. 1-10, 2018. 